\title{
Hypereosinophilic Syndrome Mimicking COVID-19 Disease
}

\author{
Pembe Derin Oygar ${ }^{1}$, Berna Oguz ${ }^{1}$, mustafa cemaloğlu ${ }^{1}$, tekin aksu $^{1}$, and yasemin \\ Ozsurekci $^{1}$ \\ ${ }^{1}$ Hacettepe University Faculty of Medicine
}

September 16, 2020

To the editor:

Hypereosinophilic syndrome is a diverse group of disorders with eosinophilia and end-organ damage. Any organ system may be involved, cardiopulmonary and neurologic diseases being the most common causes of morbidity and mortality ${ }^{1}$. HES is more commonly seen in adults but has been reported in pediatric population as well. Formerly pediatric patients who present with marked eosinophilia $(1500 / \mathrm{mm} 3)$ for at least 6 months' duration without other known causes of eosinophilia and with evidence of organ involvement used to be diagnosed as HES. Recently the International Cooperative Working Group on Eosinophil Disorders (ICOG-EO) defined HES as blood hypereosinophilia (HE) or tissue HE associated with HE-related organ damage irrespective of time (precluding the absence of an alternative diagnosis for the organ dysfunction) ${ }^{2}$ . 2019 ended with the emergence of a novel coronavirus, SARS-Co-V2, leading to a pandemic with a high attack rate causing COVID-19 disease. Since the beginning of pandemic pulmonary findings defined for COVID-19 are considered to be pathognomonic hence, chest tomography is suggested to be superior to polymerase chain reaction tests in the diagnosis of adult COVID-19 patients ${ }^{3}$.

16 -year- old female referred with the diagnosis of COVID-19 pneumonia and myocarditis. She had worsening cough for the last 10 days, initially diagnosed with pneumonia and started on antibiotics. Upon becoming dyspneic and feverish she was hospitalized. Computed tomography (CT) of the chest was reported to be suggestive of COVID-19 with bilateral peripheral consolidation areas and nodules with surrounding ground glass appearance (Figure 1a,b). Her COVID-19 PCR was negative for 3 times during this 10-day course of illness. Echocardiography revealed findings consistent with myocarditis. She was started on favipravir and intravenous immunoglobulin (IVIG) and referred to our hospital for potential extracorporeal membrane oxygenation (ECMO) requirement. On admission, her physical examination revealed tachycardia, tachypnea and hypoxia, subcostal and suprasternal retractions and bilaterally diminished breath sounds, no hepatosplenomegaly was present.

Laboratory findings were white blood cell count (WBC) $29200 / \mathrm{mm}^{3}(4100-11200)$, absolute eosinophil count (AEC) $16370 / \mathrm{mm}^{3}$ (100-500), hemoglobin (Hb) $12.5 \mathrm{~g} / \mathrm{dl}$ (11.7-15.5) , thrombocyte count $406000 /$ $\mathrm{mm}^{3}$ (159000-388000), peripheral blood smear revealed 68\% eosinophils. Cardiac enzymes were elevated; brain natriuretic peptide (BNP) $725.4 \mathrm{pg} / \mathrm{ml}$ (0-100), troponin -I $3769.5 \mathrm{ng} / \mathrm{l}$ (8.4-18.3), creatinine ki-

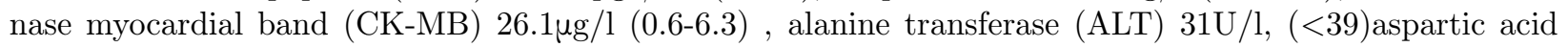
transferase (AST) $44 \mathrm{U} / \mathrm{l}(<56)$. Electrocardiogram (ECG) was at sinus rhythm, with normal PR and QT intervals. The voltages were suppressed, and there was no negative T-waves. Echocardiogram was repeated confirming myocarditis with a hypokinetic area on the apex of left ventricle. COVID PCR test repeated and found to be negative on two occasions obtained at 24 hours interval. She had been diagnosed with asthma for the last 2 years with frequent attacks and was on combined formoterol and budesonide inhaler treatment. There was no history of sinusitis or rash. Abdominal ultrasonography was normal. Stool examination revealed no parasites, there was no history of drugs. CMV, Herpes, EBV, Hepatitis B and C, Parvovirus serology and PCR were negative. Anti-neutrophil cytoplasmic antibody (ANCA) was also neg- 
ative and total Ig E was $1796 \mathrm{mg} / \mathrm{dl}$ (1.31-165), Vitamin B12 level was 227ng/L (200-590). Bone marrow aspiration revealed increased numbers of eosinophilic precursor cells (14\% eosinophilic metamyelocytes) as well as mature eosinophils (34\%) with no malignant cell and no cytogenetic abnormalities (Figure 1). Bone marrow biopsy showed a normocellular marrow with increased eosinophilic precursors, except reticulin fibrosis and mast cells. Flow cytometric evaluation showed no atypical population, JAK2V617F mutation was negative, and leukemia defining translocations including BCR/ABL1, CBFB/MYH11 were negative. Clonal hyper-eosinophilia associated rearrangements including PDGFRA, PDGFRB, FGFR1 were also negative. Upon re-evaluation of chest CT with these clinical and laboratory findings, radiology department stated that HES or Churg-Strauss should be considered as potential diagnoses as well as COVID-19 (Figure 2). CT-angiography showed no findings of vasculitis or vascular occlusion.

The patient was given high flow oxygen support, started on teicoplanin, meropenem and clarithromycin, carvedilol, enalapril and pulse methyl prednisolone treatments. On the 5th day of treatment WBC and AEC declined to $8200 / \mathrm{mm}^{3}$ and $0 / \mathrm{mm}^{3}$ respectively. Corticosteroid dose was tapered gradually; at the end of 4 weeks $\mathrm{WBC}$ and AEC were $14300 / \mathrm{mm}^{3}$ and $0 / \mathrm{mm}^{3}$ respectively. After treatment, control chest CT showed regression of lung involvement findings ( Figure 3).

Since December 2019 the whole world is struggling with a novel corona virus pandemic and COVID-19, the disease it leads to. With a high attack rate and so many deaths, it is quite understandable that COVID-19 has become number one priority diagnosis in everyday practice. The sensitivity and practicality of molecular tests has been an issue since the beginning of the pandemic and chest $\mathrm{CT}$ is claimed to be more accurate than PCR ${ }^{4}$ in the diagnosis of COVID-19 which seems to set a bias in the interpretation of chest CTs. On the other hand, whether CT is superior in diagnosis of COVID-19 in pediatric patients who experience viral pneumonia more frequently compared to adults is yet to be determined. Whether there is a pandemic or not one has to remember that the art and creativity of medicine is to evaluate every symptom and sign on a patient-based approach in order to make the correct diagnosis.

\section{References:}

1. Weller PF, Bubley GJ. The idiopathic hypereosinophilic syndrome. Blood 1994; 83:2759-79. [PubMed]

2. Simon H, Rothenberg ME, Boschner BS, et al. Refining the definition of hypereosinophilic syndrome $J$ Allergy Clin Immunol . 2010;126(1): 45-49. doi:10.1016/j.jaci.2010.03.042.

3. Xie X, Zhong Z, Zhao W, Zheng C, Wang F, Liu .CT for typical 2019-nCoV pneumonia: relationship to negative RT-PCR testing. Radiology J Chest 2020; https://doi.org/10.1148/radiol.2020200343

4. Ye, Z., Zhang, Y., Wang, Y. et al. Chest CT manifestations of new coronavirus disease 2019 (COVID-19): a pictorial review. Eur Radiol (2020). https://doi.org/10.1007/s00330- 020-068010 

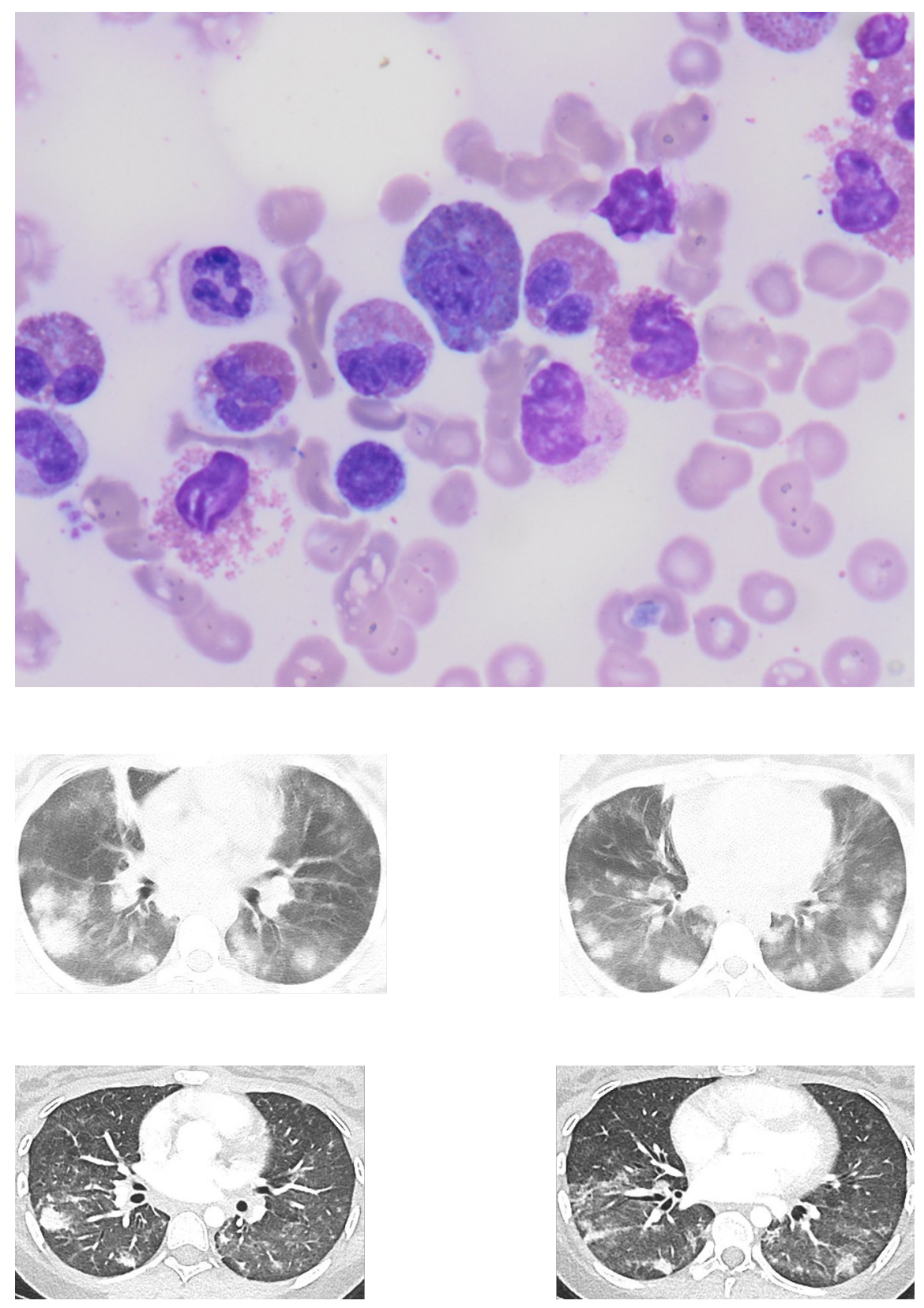